\title{
Propuesta de mejoramiento del proceso productivo en planta industrial de película stretch polivinil cloruro en Cartagena-Colombia aplicando Value Stream Mapping. Proposal to improve the production process in industrial stretch polyvinyl chloride film plant in Cartagena-Colombia applying Value Stream Mapping.
}

\author{
José Morelos-Gómez ${ }^{1}$, Eladio Enrique Peralta-Ubarnes ${ }^{2}$ \\ 1,2 Universidad de Cartagena, Cartagena - Colombia

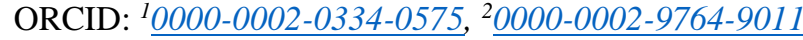

Recibido: 03 de junio de 2020.

Aprobado: 11 de noviembre de 2020.

\begin{abstract}
Resumen - Este articulo presenta la evaluación y propuesta de mejora del proceso productivo en la planta de película strech polivinil cloruro (PVC) de una empresa manufacturera en el sector industrial de Mamonal en Cartagena, Colombia. Se aplicó la metodología Value Stream Mapping (VSM), para la identificación de las familias de producción, caracterización del estado actual de los flujos de materiales e información y definición de un mapa de estado futuro. Como resultado, se logró mejoramiento en el proceso de producción en la planta de película strech, obteniendo una disminución de $42 \%$ en el tiempo de entrega requerido para los pedidos de la familia de producción de rollos para uso industrial. Con esta mejora se llega a la conclusión que, con acciones focalizadas simples, es posible lograr importantes avances en la mejora de los procesos productivos de este tipo de empresas manufactureras, siendo evidente el impacto positivo generado con el establecimiento de un flujo continuo y balanceado en la cadena de creación de valor.
\end{abstract}

Palabras Claves: value stream mapping, lean manufacturing, mejora de procesos, eficiencia operativa, flujo continuo, cadena de valor.

Abstract - This article presents the evaluation and proposal for improvement in the productive process in the plant of film polyvinyl chloride (PVC stretch) of a manufacturing company in the Mamonal industrial sector in Cartagena, Colombia. The methodology applied was Value Stream Mapping (VSM), for the identification of families of production, characterization of the present state of the flow of materials and information and definition of a future state map. As a result, improvement was achieved in the production process at the stretch film plant, obtaining a $42 \%$ decrease in the delivery time required for the orders of the roll production family for industrial use. With this improvement, the conclusion is reached that, with simple focused actions, it is possible to achieve important advances in the improvement of the productive processes of this type of manufacturing companies, being evident the positive impact generated with the establishment of a continuous and balanced flow in the value creation chain.

Keywords: value stream mapping, lean manufacturing, process improvement, operational efficiency, continuous flow, value chain. 


\section{INTRODUCCIÓN}

En un mundo globalizado, las fronteras comerciales se han venido abajo haciendo que las empresas se enfrenten constantemente a mercados más competitivos. Ya no solo las grandes multinacionales se enfrentan a otros competidores, sino que, las empresas tradicionalmente locales, se han visto abocadas a luchar por mantenerse vigentes en los mercados [1]. Según uno de los últimos informes de la Asociación Nacional de Industriales (ANDI), entre las principales dificultades que tienen los empresarios, se cuentan los altos costos y suministros de materias primas, la falta de demanda y la fuerte competencia [2], factores que influyen directamente en la competitividad y que motivan el diseño de estrategias para que las empresas logren mejores niveles de eficiencia, flexibilidad, innovación, y todo ello con los mínimos costos posibles.

En Colombia, esta situación se ha sido particularmente fuerte, dado que, la apertura económica liderada por el el Gobierno Nacional para finales de los 90's, obligó a las empresas antes resguardadas por el proteccionismo, a replantear sus estrategias corporativas [3]. En este sentido, los acuerdos de libre comercio establecidos han hecho que las empresas nacionales innoven desarrollando nuevos procesos y productos, para sobrevivir la amenaza productos importados de la competencia [4]. El escenario empeora al considerar la infraestructura del país, en la que se observan circunstancias exógenas a la empresa que generan sobrecostos que son transferidos al producto. A pesar de la estratégica posición geográfica del país y, más en concreto, de Cartagena de Indias — que le brinda una ventaja comparativa sobre otras naciones—, dicha ubicación favorable no se aprovecha a su máximo, puesto que la pobre eficiencia de los uservicios públicos, las carreteras en mal estado, los costos de transporte doméstico y otros factores, hacen que se incurra en sobrecostos que son absorbidos por el producto [5].

El escenario en la Región Caribe, no ha sido la excepción, en Cartagena, empresas ubicadas en el sector industrial de Mamonal con varias líneas de productos y de gran crecimiento, han logrado convertirse en multinacionales con presencia en varios continentes. Sin embargo, su internacionalización ha venido acompañada con grandes retos, como volverse más eficientes mediante el aumento de su productividad.

En la zona industrial de Cartagena, la planta de la empresa manufacturera objeto de estudio, tiene como propósito la fabricación de películas de polivinil cloruro (PVC), y, esta a su vez, tiene líneas de producción para película stretch, termoencogible o shrink, dispensadores caseros, moldes de aluminio e impresión de película. La línea de producción stretch, tiene una producción anual típica que corresponden al 65 \% de su capacidad instalada. El proceso consiste en la alimentación de la mezcla de materias primas a través de la extrusora y, por medio del proceso de soplado y embobinado, se obtiene la bobina o rollo, la cual pasa al proceso de empaque y acabado, quedando lista para el envío a la zona de almacenamiento y despacho.

En la actualidad, la planta de película stretch PVC presenta oportunidades de mejora en los procesos, tanto de transformación de materiales, como en el flujo de información. Sin embargo, es evidente los problemas asociados con la sobreproducción y mala calidad; con un balance de más de $30 \%$ de material no conforme en espera de reprocesar — que equivale a la producción completa de un mes-, constantes paradas no planeadas por exceso de inventario y frecuentes solicitudes de adelantos de entregas de pedidos con tiempo de espera de orden de más de 20 días. Estos inconvenientes, contribuyen al no cumplimiento en las metas de productividad y costos, al sobrepasar el costo unitario presupuestado en un 20\%, adicional, con lo que se pierden oportunidades de exportación, por no cumplir con el requerimiento de entregas. Todo lo anterior, habla de la evidente desorganización de la planta.

Es por ello, que el presente artículo, pretende establecer el nivel de eficiencia actual y las acciones necesarias para la mejora en el proceso de la planta industrial de película stretch PVC, por medio del análisis de la cadena de valor y el uso del Value Stream Mapping. Asimismo, se seleccionará una de las familias de producción para poder representar gráficamente el estado actual del flujo de información y materiales dentro de la cadena de valor, seguidamente, se identificarán los despilfarros y sus fuentes y, se establecerán las acciones requeridas para eliminarlos. Con base en estas acciones, se elaborará un mapa de estado futuro que muestre la operatividad de la organización propuesta.

\section{MARCO TEÓRICO}

\section{a. Antecedentes del lean manufacturing}

La historia de la empresa Toyota se remonta a finales del siglo XIX, cuando es fundada por Sakichi Toyoda, inspirado en un popular libro de la época que describía a un joven inventor. Inicialmente, la compañía se encargaba de la fabricación de telares bajo el nombre Toyoda, hasta que, a fines de año 1930, bajo la dirección de Kiichiro Toyoda, migró hacia la producción de vehículos de motor y asumió su nombre actual, Toyota Motor Corporation (TMC). Tras la Segunda Guerra Mundial, corriendo 1949 en un Japón que luchaba por recuperarse de la devastación y las dificultades económicas, Toyota se vio obligada a realizar ajustes en su forma de trabajar y su sistema de producción, siendo necesario la optimización del uso de todos los recursos, lo que propició un conjunto de innovaciones técnicas y de administración de la producción con miras a evitar la bancarrota [6], [7] y [8].

Considerando este propósito y la desventaja en la que estaba TMC respecto a la competencia americana, se adelantaron varias visitas a las plantas de producción de automóviles en Detroit, EE. UU. Es así como Eiji Toyoda y Taiichi Ohno, presidente de la compañía e ingeniero en jefe, respectivamente, llegaron a la conclusión de que el sistema predominante en el momento - la producción en masa- sencillamente no era aplicable a las condiciones del Japón de la postguerra. Esto se debía a que, aprovechando las economías de escala para reducir los costes de producción, la industria norteamericana estaba fabricando cantidades mayores de un número mínimo de modelos. Dicha estrategia no aplicaba a Toyota, debido al reducido tamaño del mercado de la isla nipona, el cual requería suplir las necesidades del consumidor: mayor cantidad de modelos y menores cantidades de producción [9]. En consecuencia, la optimización de costos debió cambiar de enfoque hacia una tarifa menor de fabricación, con lo que se redujo el tamaño de la operación; en otras palabras, disminuyó inventarios de productos en proceso y flexibilizó las actividades de producción para lograr un flujo continuo y responder rápidamente a la demanda [10].

De esta manera, inició una nueva configuración en el sistema de producción Toyota (TPS) con todas las herramientas que lo caracterizan, y que finalmente, se conocería como Lean Manufacturing o Lean Production. Esta filosofía se enfocó en la eliminación de despilfarros y en el aprovechamiento total de los recursos para la creación valor real requerido por el cliente [7]. Desde entonces, Toyota fue creando su propio modelo de negocio, que mostró su efectividad durante la crisis desencadenada en 1973, por el embargo árabe del petróleo, durante el cual la 
automotriz japonesa mantuvo su crecimiento mientras sus pares caían en ventas y producción [11] y [12]. En 1984, un grupo de investigadores del Massachusetts Institute of Technology (MIT) se propuso iniciar un ambicioso estudio: entender cómo la industria automotriz japonesa superó la crisis del mercado a fines de los 70, de mejor manera que la del resto del mundo. Fue entonces, cuando se puso en marcha el Programa Internacional de Vehículos de Motor (PIVM) [8]. Y durante cinco años se desarrolló una investigación exhaustiva, la cual fue financiada por empresas del sector y varios gobiernos, e incluyó entrevistas a numerosos ejecutivos de las corporaciones fabricantes de automóviles y visitas a más de noventa plantas de montaje en 17 países. El resultado de estos estudios dio lugar a la publicación de la primera edición del libro La máquina que cambió al mundo en año 2017, manifiesto que introdujo en la escena mundial el término Lean Manufacturing (LM), como la razón por la que las empresas japonesas habían aumentado, de forma consistente, su participación en el mercado de vehículos, incluso cuando el sector se encontraba en crisis [13] y [14].

\section{b. Principios de la filosofía lean manufacturing}

La primera edición de Lean Thinking en 1996, se presenta como la guía para la aplicación de la manufactura Esbelta en cualquier industria, en cual, Womack y Jones [12] y [15] explican en detalle la filosofía detrás del modelo, condensándola en cinco principios:

Definición de valor: corresponde a los atributos deseados que debe tener el producto o servicio para satisfacer una necesidad. Estos son definidos por el cliente.

Flujo de valor $(\mathrm{FV})$ : conjunto de actividades específicas necesarias para diseñar, solicitar y suministrar un producto específico, desde la concepción hasta su lanzamiento a fabricación, desde el pedido a su entrega, y desde las materias primas hasta que llega a manos del cliente.

Flujo continuo: se refiere a la realización, sin interrupciones ni devoluciones en el flujo, de todas las actividades necesarias para la creación de valor desde el punto de vista del producto.

Pull (atraer): bajo la filosofía Lean, la demanda es la que desencadena el proceso productivo, es el cliente quien jala para sí el producto a través de la cadena de valor. De esta manera, solo se consumen recursos y materiales en la cantidad de producto o servicio con demanda manifiesta.

Perfección: se establece la necesidad de buscar constantemente la perfección del sistema mediante el desarrollo de mejores metodologías para crear valor. Cuando se empeña en acelerar el flujo de valor, la organización encontrará obstáculos que, al ser eliminados, darán paso a nuevas dificultades antes ocultas; como consecuencia, la evaluación permanente en búsqueda de la excelencia se hace precisa.

Se destaca la aplicación universal, lo cual han demostrado ser exitosos en la creación de marcos de referencia para la implantación y aprovechamiento de las herramientas asociadas a Lean Manufacturing, en lo que tiene que ver con la mejora continua de los procesos [16] y [17]. En otro enfoque, los principios del LM pueden clasificarse desde el factor humano y desde la óptica de medidas operacionales y técnicas a usar [9] y [18]

\section{c. Lean manufacturing desde el factor humano y la manera de trabajar y pensar}

El mejoramiento de los procesos productivos en la industria, requiere del desarrollo de una cultura de creación de valor desde la perspectiva del factor humano, enfocando sus actividades en las siguientes practicas organizacionales [19]: a) trabajar en la planta y comprobar las cosas in situ, b) formar líderes de equipos que asuman el sistema y lo enseñen a otros, c) interiorizar la cultura de "parar la línea", d) crear una organización que aprenda mediante la reflexión constante y la mejora continua, e) desarrollar personas involucradas que sigan la filosofía de la empresa, f) respetar la red de suministradores y colaboradores, ayudándoles y proponiéndoles retos, g) identificar y eliminar funciones y procesos innecesarios, $h$ ) promover equipos y personas multidisciplinares, i) descentralizar la toma de decisiones, $\mathrm{j}$ ) integrar funciones y sistemas de información y k) obtener el compromiso total de la dirección con el modelo Lean.

\section{d. Lean manufacturing desde las medidas operacionales y técnicas}

Igualmente, para responder a la mejora de los procesos productivos se requiere la aplicación de buenas prácticas de manufactura, uso adecuado de herramientas y procedimientos en los procesos operacionales, que conduzcan al logro de resultados con eficiencia y calidad [20], así: a) crear un flujo de proceso continuo que visualice los problemas en la superficie, b) utilizar sistemas pull para evitar la sobreproducción, c) nivelar la carga de trabajo para equilibrar las líneas de producción, d) estandarizar las tareas para implementar la mejora permanente, e) utilizar el control visual para la detección de problemas, f) eliminar inventarios a través de las diferentes técnicas "justo a tiempo" (JIT), g) reducir los ciclos de fabricación y diseño y h) conseguir la eliminación de defectos.

Por su parte, Taiichi Ohno, principal gestor del sistema de producción Toyota, define la filosofía como la búsqueda de "la eliminación absoluta del excedente", y lo soporta en dos pilares fundamentales [21]:

Justo a tiempo: se refiere al suministro de cada parte o componente en el momento y la cantidad justa que necesita el proceso. El logro completo de esto supondría la eliminación de los inventarios en proceso y en espera de ingresar a este, aportando a la rentabilidad; así como el incremento de la productividad al alcanzar el flujo continuo [22]. Una línea de producción que se basa en la previsión de las cantidades a usar tiende a programar cada uno de los procesos de manera independiente, fabricando según estos y sin tener en cuenta los procesos aguas abajo. Cualquier variación que afecte la ejecución del plan de producción, resultará en una cantidad de inventarios, sin necesidad inmediata que ya ha consumido recursos.

Automatización con un toque humano o autonomación: se diferencia de la automatización en que se adicionan a las máquinas sistemas capaces de detectar fallos en el proceso y parar la línea inmediatamente, previniendo la fabricación de productos defectuosos. De esta forma, el enfoque de la producción se modifica con el papel del operador, toda vez que este último, pasa de ser quien manipula permanentemente la máquina a intervenir solo cuando el proceso se detiene por algún problema específico; así, es posible que un solo operador atienda diferentes máquinas y que varias personas, puedan intervenir en el evento de alguna falla y apoyar en la solución del mismo [23]. 


\section{e. Los despilfarros o mudas}

Como se ha indicado, la filosofía Lean es la búsqueda de la eliminación de todo tipo de despilfarros existentes en el proceso, los cuales se definen como cada actividad que consuma recursos sin agregar valor. La palabra japonesa usada es muda y Taiichi Ohno, clasificó estas mudas en siete tipos principales, como se muestra a continuación [6], [12] y [24].

Defectos: constituyen muda por el trabajo adicional que se genera como consecuencia de no realizar la tarea de forma correcta desde la primera vez, obligando a la reposición de material e incrementando el inventario de material no conforme o el reproceso de éstos [25].

Sobreproducción: la fabricación de más de lo que se requiere, o la inversión en la compra y/o diseño de equipos con mayor capacidad de la necesaria. El problema con esta condición es que oculta las verdaderas ineficiencias del sistema entretanto que se consumen recursos, tiempo, material y esfuerzo en productos, que terminarán en el almacén como inventario que no es monetizado inmediatamente [26].

Existencias (inventarios): forma más clara e importante de muda ya que esconde todas las demás formas de despilfarro en la compañía y, como resultado, la necesidad de encontrarles solución se diluye [6], [10], [27] y [28].

Procesamiento innecesario: cualquier proceso que se le aplique al producto y que no genere más valor del que el cliente está dispuesto a pagar, se concibe como despilfarro [29].

Transporte (de productos) no necesarios: productos en proceso deben pasar desde una estación de trabajo en forma directa, con el menor recorrido posible y sin ningún tipo de espera. Cuando no se cumplen estas condiciones se genera un sobrecosto, el cual se manifiesta en un desplazamiento adicional al requerido, tiempo de procesamiento y riesgos de daño. Su causa es, por lo general, el mal diseño de la planta física y la disposición de las máquinas y estaciones de trabajo [30].

Movimientos (de personas) no necesarios: flujo de personal con desplazamientos más largos y frecuentes que el necesario. Sus causas son similares a las del despilfarro tipo transporte [31].

Esperas: tiempo perdido tras una secuencia de trabajo o proceso ineficiente. Se debe a la mala coordinación entre las diferentes actividades que conforman la cadena de valor [10] y [32].

La identificación y ubicación de las mudas dentro de la cadena de valor es el primer paso para la eliminación de los mismos, optimizando el proceso y los resultados de la compañía [28]. En torno a esta idea, se han desarrollado herramientas y metodologías para facilitar la evaluación constante del flujo del valor, y es allí donde cobra importancia el desarrollo del Value Stream Mapping [33].

\section{f. Modelos de mejoramiento de procesos}

La necesidad de mejorar los procesos ha sido manifiesta desde el inicio de la era industrial, no obstante, el enfoque con que se aborda ha ido cambiando a medida que se suceden los distintos paradigmas de modelos organizacionales [34]. A lo largo del siglo XX, se han desarrollado metodologías que buscan facilitar la tarea de elaborar un diagnóstico del proceso para el cual se desea la mejora, en búsqueda de reducir los tiempos de respuesta y costos de operación mediante la eliminación de despilfarros [35]. Definir el Flujo de Valor (FV) y asegurar que sea continuo resulta clave para lograr la optimización del sistema de producción completo, lo que permite definir las actividades para la creación de valor y que este se dirija hacia al cliente. En este proceso se deben identificar con claridad las actividades que generan valor, y aquellas que no, para actuar en concordancia [12] y [36].

En este sentido, la filosofía Lean Manufacturing ha desarrollado diferentes herramientas, y en particular, la identificación del flujo de valor, mediante la herramienta de mapeo del flujo de valor o Value Stream Mapping (VSM), que consiste en la elaboración de un diagrama con las actividades que aportan a la obtención del producto final que recibe el cliente; esto se realiza desde el enfoque de transformación de materiales y desde el flujo de información [37], [38] y [39]. El VSM fue aplicada en la Toyota Motor Corporation, para facilitar la visión global de los procesos y hallar las fuentes de despilfarros y oportunidades de perfeccionar la creación de valor [40]. Previo a su desarrollo, existían diversas técnicas que no aportaban de forma integral al análisis de la cadena de valor, ni a la eliminación de la totalidad de los tipos de despilfarros identificados por el LM [41].

Es así como, variados estudios en donde se ha desarrollado el VSM destacan que es una técnica globalmente reconocida, y sus aplicaciones no solo se limitan al sector manufacturero, es decir, su uso se ha extendido a diferentes tipos de organizaciones y campos, tales como, la aplicación en talleres, hospitales, empresas de tecnología e información (IT) y de publicidad y otros servicios, lo que arroja beneficios en todos los casos, representados en aumento de la productividad y del nivel de servicio [42], [43], [44] y [45]

\section{g. Herramientas para la eliminación de despilfarros}

Los modelos de mejoramiento de proceso muestran un diagnóstico de la cadena de valor que se complementa con otras técnicas para la eliminación de despilfarros; estas pueden clasificarse como herramientas operativas [46] útiles en la aplicación de sus principios y que pueden ser implementadas en múltiples entornos de forma individual, o como parte de una estrategia global. A continuación, se listan algunas de estas técnicas [47]:

- Cinco S (5s): esta herramienta tiene como objetivo la creación de lugares de trabajo más organizados, limpios y seguros, a través de la aplicación de cinco conceptos clave: separar (seiri), ordenar e identificar (seiton), limpieza (seiso), estandarizar (seiketsu) y disciplina (shitsuke). Más que una aplicación específica, esta técnica busca crear cultura entre los trabajadores, manteniendo los resultados en el tiempo [48] y [49].

- SMED: técnica para reducir los tiempos de cambio entre productos. Adopta la identificación y delimitación de las actividades requeridas, toma acciones en cada una de ellas en búsqueda de la eliminación de las tareas innecesarias, las clasifica entre externas 
(aquellas que para su ejecución no requieren de la máquina detenida) e internas, y las optimiza. Con esta técnica se busca tiempos de cambio mínimos que ronde la duración de un minuto por evento [50] y [51].

- Heijunca: técnica empleada para nivelar la producción en entornos de múltiples productos. Basada en la demanda durante un rango de tiempo establecido, se trata de la creación mezclas de producción estables para el suministro constante de los productos en lapsos pequeños; usualmente un turno de trabajo para lograr una programación previsible y suavizada [52] y [53].

- Jidoka: capacidad que debe tener el operador, o el sistema de la máquina, de parar la línea de producción cuando se detecten defectos. Esto con el fin de atacar y corregir el problema sin continuar fabricando material no conforme. Tiene que ver con el concepto de autonomación, esbozado por Ohnno, según el que la máquina debe tener sistemas capaces de frenar la operación una vez ocurra un fallo que afecte la calidad del producto [54].

- Mantenimiento productivo total (TPM): conjunto de actividades que buscan empoderar a los operadores de producción en el mantenimiento de sus equipos, al detectar y reparar componentes defectuosos. En trabajo conjunto con el área de mantenimiento, se busca la mejora en la disponibilidad de las máquinas eliminando las pérdidas por fallas [55].

- Kanban: sistema de comunicación entre procesos que usa señales o tarjetas para la sincronización y control de la producción o suministro de piezas. Al ser retiradas para consumo, estas piezas desencadenan el proceso de abastecimiento necesario. Esta herramienta permite la eliminación de programas de producción independientes para cada proceso en la búsqueda de un flujo continuo [56] y [57].

\section{h. Value Stream Mapping}

La cadena de valor es definida como el conjunto de acciones que se necesitan para mover un producto a través de los flujos esenciales, a saber: la producción, que representa la transformación física del producto, desde que es materia prima hasta que le llega a las manos del cliente, y, el flujo de diseño, que parte de la concepción del producto hasta su lanzamiento. Dentro del flujo de producción es posible, a su vez, identificar dos flujos distintos. Los materiales, que se transforma, y la información, que lleva las instrucciones y retroalimentaciones entre los diferentes procesos [26]. El VSM se define como una herramienta de diagnóstico que representa en forma gráfica las acciones contenidas en el flujo de producción, relacionando cada una de las actividades por las que atraviesa el producto, y siguiendo el tránsito desde el cliente hasta el proveedor de la materia prima [58] y [59]. El resultado de esta cartografía es un diagrama detallado de las actividades que componen la cadena de valor, lo que permite ver y cuestionar el proceso y los despilfarros actuales con sus fuentes, facilitando así el establecimiento de una visión del estado futuro del proceso una vez eliminados [60].

Para Rohac y Januska, el VSM descubre numerosos problemas y cuellos de botella en la operación, así como muestra los despilfarros y sus fuentes, permitiendo luego — con ayuda de otras técnicas, como los cinco por qué o el Diagrama de Ishikawa—identificar las causas de estos, y así trazar, las estrategias para su solución definitiva a problemas, sin embargo, destacan la imposibilidad de dibujar en simultáneo todas las líneas de producto dentro de una compañía, lo que obliga realizar un análisis independiente de cada una de ellas, lo que podría resultar complejo para empresas con un portafolio amplio que centralizan servicios para la producción [61]. Asimismo, el VSM no detalla el consumo de tiempo que demanda el comportamiento dinámico de los procesos de producción [62].

\section{METODOLOGÍA O PROCEDIMIENTOS}

Este articulo de investigación, es de tipo descriptivo y explicativo, dado la naturaleza y alcance del estudio. Como fuentes de información primaria se consideraron los datos derivados de la observación directa de los procesos productivitos de la planta industrial de película strech de producción de rollos de polivinil cloruro. Las fuentes secundarias utilizadas se relacionaron con los libros, tesis y artículos científicos de bases datos indexadas en Scopus, Web of Science (WoS) y Science Direct, relacionados con la aplicación de la metodología VSM, el cual facilitó la revisión y selección de 50 artículos relacionados con el tema de estudio.

La aplicación in Situ de la técnica de mejoramiento VSM, permitió a partir del análisis racional, lógico y técnico de la secuencia del proceso productivo, identificar y describir las fuentes de despilfarro e ineficiencias en el proceso productivo, explicando las causas y variables criticas del mismo, con lo cual se logró definir las acciones estratégicas en beneficio de la optimización de los procesos de la planta strech PVC. Para la evaluación proceso productivo en la planta industrial de película strech PVC, se estructuraron las siguientes seis fases, con el propósito de ajustar las acciones correctivas de conformidad con lo establecido en la metodología de flujo de valor asi:

Fase 1: Recopilación y sistematización de la información teórica que soporta la aplicación de la propuesta considerando material científico de revistas internacionales indexadas en base de datos como Scopus, Web of Science (WoS) y Science Direct, así como los anales del modelo VSM y casos de implementación en otras empresas internacionales similares del sector industrial manufacturero.

Fase 2. Clasificación por grupos o familias de los artículos fabricados en la planta industrial PVC, con base en los procesos y máquinas comunes entre ellos. Se consideró la elección de un grupo para aplicar la metodología VSM.

Fase 3. Esquematización del mapa de flujo de valor actual, para la familia de productos seleccionada. Se describirá cada una de las actividades requeridas para que el producto obtenga el valor requerido por el cliente final. Se relacionarán los datos suficientes para una comprensión sistemática del flujo de valor.

- Fase 4. Identificación y análisis de los despilfarros o mudas dentro del flujo de valor actual.

- Fase 5. Mapeo de la situación futura o deseable que muestra el proceso tras aplicar las mejoras definidas en la fase anterior.

- Fase 6. Elaboración de la propuesta para la aplicación de las mejoras definidas en la Fase 5. 


\section{RESULTADOS ANÁLISIS E INTERPRETACIÓN}

\section{Aplicación del VSM en planta strech PVC}

Para la aplicación de la herramienta en la planta strech PVC de la empresa manufacturera objeto de estudio, se utilizó el siguiente flujograma de creación de valor (ver figura 1), el cual permitió identificar las oportunidades de mejora dentro del proceso y seleccionar las herramientas LM que mejor adaptación presentaron al proceso productivo a optimizar.

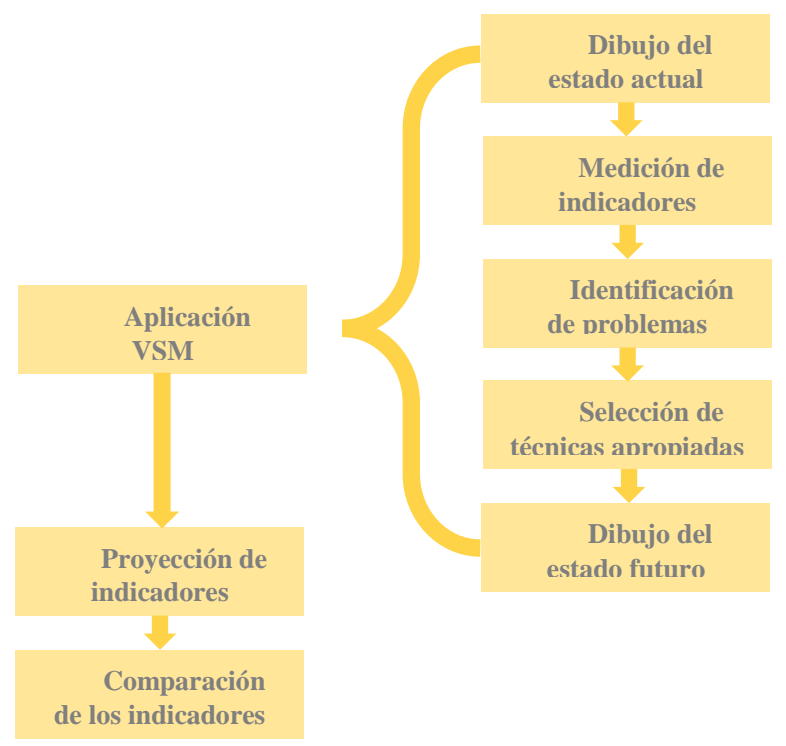

Figura 1: Flujograma de implementación VSM.

Fuente: Elaboración propia.

\section{a. Caracterización del estado actual del proceso - Mapa de estado actual}

La empresa industrial manufacturera objeto de investigación, centra sus actividades en la producción y comercialización de artículos fabricados en materiales plásticos, especiales para las áreas de construcción, decoración y empaque de alimentos. Entre los productos que ofrece la división de empaques de alimentos se encuentran las películas tipo stretch, fabricados en la planta de película de PVC, con una producción anual cercana a las 6,000 toneladas.

El proceso consta de la extrusión de la resina de PVC junto con los plastificantes y aditivos necesarios para fabricar compuesto en forma de pellets. Estos son almacenados hasta su uso, en un subproceso posterior de extrusión en forma de película soplada que se embobina en tubos de cartón, según las distintas medidas requeridas para cada familia de productos. Las bobinas resultantes pasan por procesos de maduración, acabado y/o empaque, dependiendo de la familia de producción que se esté elaborando.

El modelo de producción se basa en lotes de producto, con base en los niveles objetivos de inventario, suavizados con las restricciones acordadas con el departamento de Producción (establecimiento de lotes mínimos). La empresa califica sus productos en dos tipos: i) de línea y ii) especiales; los primeros, son aquellos que se mantienen con un nivel de inventario adecuado para cubrir la demanda por un periodo específico, con la idea de satisfacer los pedidos de los clientes de manera inmediata; y los segundos o (make to order), que son productos que solo se fabrican en el momento de ingresar un pedido, por lo que no se mantiene inventario de ellos.

El proceso de producción inicia con la solicitud de pedido del cliente, la cual es comparada con las existencias de productos terminados en inventarios, con el propósito de conocer faltante que habría que suplir para cubrir el pedido y/o mantener el nivel de inventario deseado. En el caso de los productos especiales, el inventario deseado es cero, de manera que se envía la señal a área de producción, por medio de una orden que incluye el Stock Keeping Unit (siglas en inglés SKU), la cantidad y fecha requerida, como se muestra en figura 2. El departamento de producción realiza la requisición de materiales y el respectivo balance de inventarios de los componentes, para solicitar los faltantes al departamento de compras (suministros). Una vez se tenga la confirmación de los recursos necesarios para la producción (maquinas, personas y materiales), se procede con la programación de la producción, asignando para cada orden de producción una máquina y una secuencia que se define en el programa de producción.

$$
\begin{aligned}
& \text { Orden de producción }=\text { Pedidos }- \text { Inventario } \quad \text { Ec. } 1 \\
& \text { Orden de producción }=(\text { pedido }- \text { inventario })+\text { venta promedio mensual } * \text { nivel óptimo de invent. }
\end{aligned}
$$

El producto terminado es dejado a disposición del departamento de logística, que se encarga de la recepción y acopio de los SKU mediante recogidas diarias programadas y, seguido, realiza la expedición a los clientes finales. 


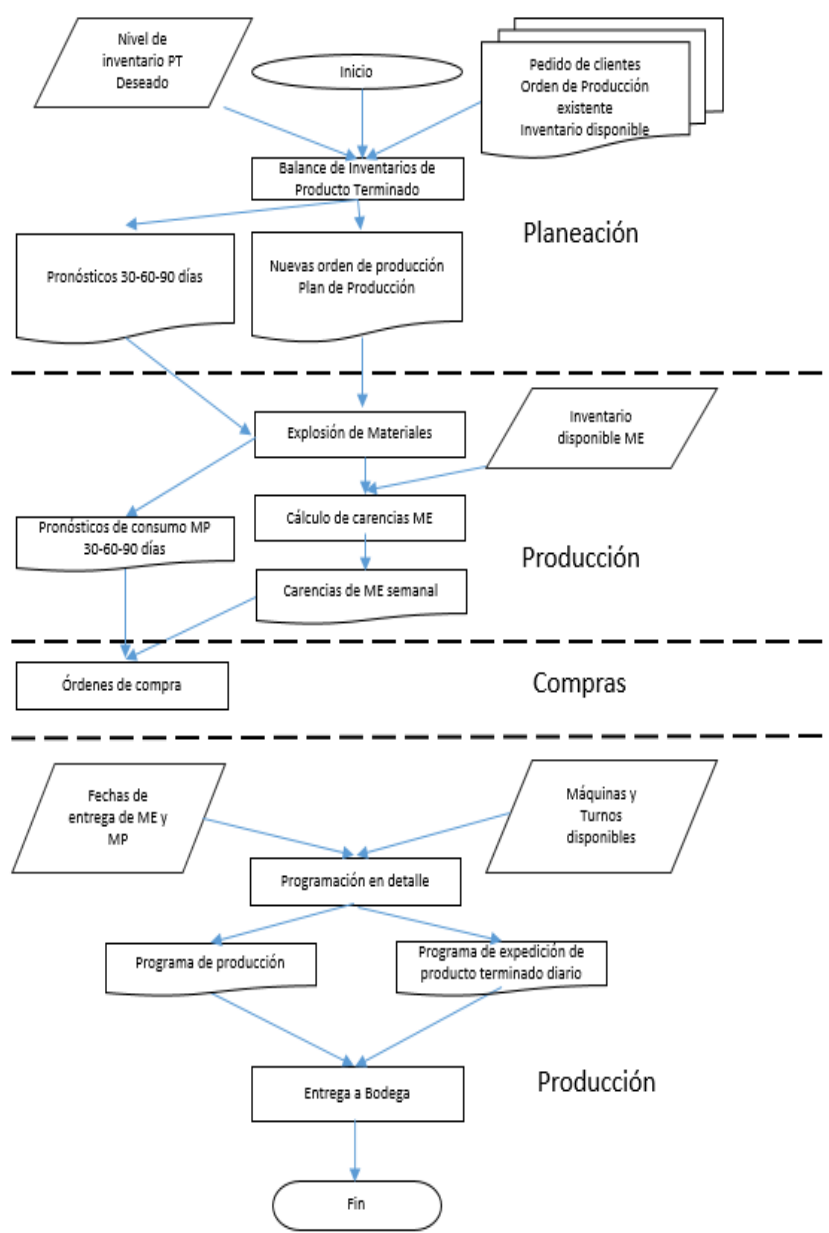

Figura 2: Proceso de programación de la producción planta stretch PVC. Fuente: Elaboraci propia.

\section{b. Selección familia de productos}

Las películas de stretch PVC tienen la característica de ser muy versátiles. Su uso es ajustable en diversas finalidades como el embalaje de carga, empaque en lavanderías, embalaje de productos de consumo industrial y empaque de alimentos. Para esta última, las aplicaciones pueden pasar desde el empaque industrial en máquinas automáticas, uso manual, dispensadores caseros y para catering. El proceso de transformación es similar para todas las familias de producto. Son comunes las actividades iniciales, diferenciándose hacia las etapas de acabado y empaque de los rollos. En cada familia los SKU atraviesan exactamente por el mismo proceso, en el que solo se dirigen por variaciones en las dimensiones específicas de cada producto e información que se muestra en el empaque y etiquetado; con ello se crea una clasificación adicional desde el enfoque comercial, irrelevante para el análisis de proceso, y referido a marca y líneas de producto. Desde el enfoque de producción, se distinguen las siguientes tres familias de producto:

1. Dispensadores: bobinas fabricadas en espesores bajos — entre 8 y 10 micras - y metrajes entre 300 y 914 m. Se suministran con empaque y accesorios con función de facilitar su uso al dispensar la cantidad de película requerida. Es un diseño para utilización manual, casera y de catering -abastecimiento-.

2. Rollos de uso industrial: Incluye rollos con metrajes mayores a 1,000 $\mathrm{m}$ y con acabado pulido para facilitar el desembobinado en procesos industriales, ya sea para aplicación manual o en máquina automática.

3. Rollos madre: bobinas que constituyen materia prima para procesos adicionales de rebobinado y/o impresión de la película, con metrajes mayores a 4,500 m y sin empaque individual. En tabla 1, se muestra la clasificación de los productos entre las familias de producción identificadas:

Tabla 1: Productos y su clasificación en familias por procesos.

\begin{tabular}{|c|c|c|c|c|c|c|c|}
\hline & \multicolumn{6}{|c|}{ Actividades } & \\
\hline Productos & $\begin{array}{l}\text { Dosificación } \\
\text { y mezclado }\end{array}$ & Peletizado & $\begin{array}{l}\text { Extrusión y } \\
\text { embobinado }\end{array}$ & $\begin{array}{l}\text { Refilado y } \\
\text { empaque }\end{array}$ & $\begin{array}{l}\text { Armado de } \\
\text { caja y sellado }\end{array}$ & Paletizado & Familias \\
\hline Antibacterial Cutter box & $\mathrm{x}$ & $\mathrm{x}$ & $\mathrm{x}$ & & $\mathrm{x}$ & $\mathrm{x}$ & \multirow{3}{*}{ Dispensadores } \\
\hline $\begin{array}{l}\text { Pro Cling Film for cutter } \\
\text { box USA }\end{array}$ & $\mathrm{x}$ & $\mathrm{x}$ & $\mathrm{x}$ & & $\mathrm{x}$ & $\mathrm{x}$ & \\
\hline $\begin{array}{l}\text { Standard Cling Film for } \\
\text { cutter box LATAM }\end{array}$ & $\mathrm{x}$ & $\mathrm{x}$ & $\mathrm{x}$ & & $\mathrm{x}$ & $\mathrm{x}$ & \\
\hline
\end{tabular}




\begin{tabular}{|c|c|c|c|c|c|c|c|}
\hline Envol & $\mathrm{x}$ & $\mathrm{x}$ & $\mathrm{x}$ & & $\mathrm{x}$ & $\mathrm{x}$ & \\
\hline $\begin{array}{l}\text { Darnel IL (Double layer) } \\
\text { Automatic Wrapper }\end{array}$ & $\mathrm{x}$ & $\mathrm{x}$ & $\mathrm{x}$ & $\mathrm{x}$ & & $\mathrm{x}$ & \multirow{13}{*}{$\begin{array}{l}\text { Rollos de uso } \\
\text { industrial }\end{array}$} \\
\hline Duplo & $\mathrm{x}$ & $\mathrm{x}$ & $\mathrm{x}$ & $\mathrm{x}$ & & $\mathrm{x}$ & \\
\hline Digi Wrap & $\mathrm{x}$ & $\mathrm{x}$ & $\mathrm{x}$ & $\mathrm{x}$ & & $\mathrm{x}$ & \\
\hline $\begin{array}{l}\text { General Purpose Manual } \\
\text { Wrap }\end{array}$ & $\mathrm{X}$ & $\mathrm{x}$ & $\mathrm{x}$ & $\mathrm{x}$ & & $\mathrm{x}$ & \\
\hline $\begin{array}{l}\text { Manual \& Automatic } \\
\text { Mushroom Wrap }\end{array}$ & $\mathrm{x}$ & $\mathrm{x}$ & $\mathrm{x}$ & $\mathrm{x}$ & & $\mathrm{x}$ & \\
\hline $\begin{array}{l}\text { Meat Wrap/Automatic } \\
\text { Omori \& Ulma Wrapper }\end{array}$ & $\mathrm{x}$ & $\mathrm{x}$ & $\mathrm{x}$ & $\mathrm{x}$ & & $\mathrm{x}$ & \\
\hline $\begin{array}{l}\text { Meat Wrap/Automatic } \\
\text { Wrapper }\end{array}$ & $\mathrm{x}$ & $\mathrm{x}$ & $\mathrm{x}$ & $\mathrm{x}$ & & $\mathrm{x}$ & \\
\hline $\begin{array}{l}\text { Meat Wrap/Manual } \\
\text { Wrapper }\end{array}$ & $\mathrm{x}$ & $\mathrm{x}$ & $\mathrm{x}$ & $\mathrm{x}$ & & $\mathrm{x}$ & \\
\hline $\begin{array}{l}\text { Produce Wrap/Manual } \\
\text { \& Automatic Wrapper }\end{array}$ & $\mathrm{X}$ & $\mathrm{x}$ & $\mathrm{x}$ & $\mathrm{x}$ & & $\mathrm{x}$ & \\
\hline $\begin{array}{l}\text { Blue-Green Laundry } \\
\text { Wrap }\end{array}$ & $\mathrm{X}$ & $\mathrm{x}$ & $\mathrm{x}$ & $\mathrm{x}$ & & $\mathrm{X}$ & \\
\hline Clear Laundry Wrap & $\mathrm{x}$ & $\mathrm{x}$ & $\mathrm{x}$ & $\mathrm{x}$ & & $\mathrm{x}$ & \\
\hline Blue Meat Film & $\mathrm{X}$ & $\mathrm{x}$ & $\mathrm{x}$ & $\mathrm{x}$ & & $\mathrm{x}$ & \\
\hline $\begin{array}{l}\text { Meat Wrap/Automatic } \\
\text { Ishida Wrapper }\end{array}$ & $\mathrm{x}$ & $\mathrm{x}$ & $\mathrm{x}$ & $\mathrm{x}$ & & $\mathrm{X}$ & \\
\hline $\begin{array}{l}\text { Bobinas madre para } \\
\text { impresión }\end{array}$ & $\mathrm{x}$ & $\mathrm{x}$ & $\mathrm{x}$ & $\mathrm{x}$ & & & \multirow{2}{*}{ Rollo madre } \\
\hline $\begin{array}{l}\text { Bobinas madre para } \\
\text { rebobinado }\end{array}$ & $\mathrm{x}$ & $\mathrm{x}$ & $\mathrm{X}$ & $\mathrm{x}$ & & & \\
\hline
\end{tabular}

Fuente: Elaboración Propia.

Para la selección de la familia de productos, se utilizó el análisis racional y lógico de la distribución de toneladas fabricadas, considerando la familia de producto que más impacte en los costos de producción así (ver tabla 2):

Tabla 2. Distribución de Kg. Fabricados por familias de producto.

\begin{tabular}{|l|c|c|}
\hline \multicolumn{1}{|c|}{$\begin{array}{c}\text { Familia } \\
\text { productos }\end{array}$} & Producción(kg) & Total (\%) \\
\hline Dispensadores & $1,169,831$ & 20 \\
\hline Rollo madre & 487,641 & 9 \\
\hline $\begin{array}{l}\text { Rollo de uso } \\
\text { Industrial }\end{array}$ & $4,064,215$ & 71 \\
\hline $\begin{array}{l}\text { Total película } \\
\text { strech PVC }\end{array}$ & $5,721,687$ & 100 \\
\hline
\end{tabular}

Fuente: Elaboración propía

De acuerdo con el análisis mostrado en la figura 3, la familia de producto a mapear será la de los rollos para uso industrial, toda vez que la fabricación de esta familia de productos corresponde al $71 \%$ del total de kilogramos producidos por la planta en año 2019, lo que representó un mayor impacto en términos de los costos de producción.

\section{c. Recolección de datos del flujo de valor}

Para la elaboración del mapa actual de la familia de producción Rollos Para Uso Industrial (RPUI), se realizó la recolección de los datos a seguir considerando las actividades del proceso de tabla 1.

1. Dosificación y mezclado: en este proceso, y de acuerdo con recetas, se dosifican las cantidades exactas de PVC, plastificantes, y aditivos líquidos y sólidos, para luego mezclarse a unas condiciones dadas de temperatura del batch y, finalmente, enfriarse para formar una mezcla de compuesto seco. Tiene dos líneas de producción:

- Equipo: línea de producción automática para control de batch de cargue de materias primas, dosificación, pesaje, mezclado y enfriado del compuesto.

- Tiempo de ciclo: cada batch dura 15 minutos en promedio para hacer todo el proceso. La capacidad del sistema es de 2,400 $\mathrm{kg}$ por hora compartida, asignando el $71 \%$ para la familia de productos en evaluación, con un promedio de $7.13 \mathrm{~kg}$ por rollo. Es decir, tenemos un tiempo de ciclo de 0.25 minutos por rollo.

- Tiempo de cambio de productos: el tiempo promedio de duración de cambios de referencia es de 15 minutos.

- Fiabilidad: en los históricos cuando este sistema ha corrido la familia de productos en evaluación ha tenido una disponibilidad promedio de $95 \%$. 
- Operarios: en este proceso se utiliza un operador para monitorear y manipular el sistema de control automático de la línea de producción de mezclado y el sistema de control automático del proceso de pelletizado, y hacer ajustes operativos, varios requeridos por ambos procesos. Se estima que su tiempo está compartido entre esas tres actividades en un 70 \%-20\%-10\%, respectivamente.

- Inventarios observados: una hora de compuesto en la tolva de transferencia, esto es aproximadamente $2,400 \mathrm{~kg}$ de compuesto seco.

- Estaciones: para correr esta familia de producto se pueden usar cualquiera de las dos líneas de producción disponibles.

2. Pelletizado: la mezcla de compuesto seco pasa ahora a la extrusora, donde se convierte en pellets esféricos sólidos. Tiene dos líneas de producción.

- Equipo: línea de producción automática para cargue del compuesto seco, dosificación, extrusión, pelletizado y desgranado del pellet.

- Tiempo de ciclo: proceso continuo con capacidad de $2,085 \mathrm{~kg}$ por hora, a la que se le asigna el $71 \%$ para la familia de productos en evaluación, con un promedio de $7.13 \mathrm{~kg}$ por rollo. Es decir, un tiempo de ciclo de 0.3 minutos por rollo.

- Tiempo de cambio de productos: el tiempo promedio de duración de cambios de referencia es de 15 minutos.

- Fiabilidad: en los históricos, cuando este sistema ha corrido la familia de productos en evaluación, ha tenido una disponibilidad promedio de $95 \%$.

- Operarios: este proceso comparte el mismo operador del proceso anterior. Es decir, se utiliza un operador para monitorear y manipular el sistema de control automático de la línea de producción de mezclado y el sistema de control automático del proceso de pelletizado, y hacer ajustes operativos varios requeridos por ambos procesos. Se estima que su tiempo está compartido entre estas tres actividades en un 70\%-20\%-10\% respectivamente. Sin embargo, el proceso de pelletizado tiene un operador exclusivo al final de la línea de producción para colocar y monitorear el llenado, movilizar y pesar los big bags de pellets.

- Inventarios observados: tres (3) días de consumo continuo de pellets por el área de extrusión-embobinado.

- Estaciones: Para correr esta familia de producto se pueden usar cualquiera de las dos líneas de producción disponibles, que se comparten con las demás familias de producto.

3. Extrusión/embobinado: En este proceso se extruyen los pellets, obtenidos del proceso anterior, para convertirlos en una burbuja plástica soplada de forma tubular hueca, que recibe la embobinadora para aplanarla y formar así dos capas. Estas capas luego se dividen para irse a su respectiva estación, donde pasan por un proceso de tensionado mediante rodillos; en ellos, se va formando el rollo de la referencia de película requerida. Tiene seis líneas de producción.

- Equipo: línea de producción automática de cargue del pellet, dosificación, extrusión de burbuja o película soplada y embobinado doble estación de esta.

- Tiempo de ciclo: para la familia de productos en evaluación se tiene una capacidad promedio de 72.65 rollos por hora, es decir que el tiempo de ciclo de 0.8 minutos por rollo.

- Tiempo de cambio de productos: el tiempo promedio de duración de cambios entre referencias es de diez minutos.

- Fiabilidad: en los históricos, cuando este sistema ha corrido la familia de productos en evaluación, ha tenido una disponibilidad promedio de $94 \%$.

- Operarios: en este proceso, un operador es el encargado de parametrizar el proceso de dosificación y extrusión, controlar la calidad de la burbuja soplada y ajustar la embobinadora en los arranques de la línea. Luego se concentra en el proceso de alistamiento de cores en los ejes y retira los rollos de película embobinados una vez finalizados los ciclos. Solo en algunos casos, como caída de burbuja u otro tipo de situaciones de parada críticas, se recibe apoyo de un segundo operador.

- Inventarios observados: tres días que todos los rollos que salen de este proceso duran en la zona de maduración antes de pasar a otro proceso.

- Estaciones: la planta cuenta con seis estaciones disponibles, sin embargo, normalmente permanecen solo cuatro máquinas en trabajo, las cuales se comparten con las demás familias de producto. Las máquinas cinco y seis entran en operación en temporada alta y eventos atípicos de incrementos puntuales de ventas.

4. Refilado y empaque: tras ser embobinados, los rollos pasan a este proceso para darle acabado a los bordes. Son cuatro máquinas. En la familia de producto Rollos Para Uso Industria, el producto se empaca en cajas individuales.

- Equipo: máquina semiautomática para darle acabado a los bordes de los rollos de película en un tiempo de cuarenta segundos por rollo.

- Tiempo de ciclo: todas las actividades comprendidas entre recoger pallet en la zona de maduración, refilar, empacar y preparar pallet de producto terminado. Estas incluyen diversos ajustes y movimientos operativos, y nos da un tiempo de ciclo de un minuto por rollo.

- Tiempo de cambio de productos: el tiempo promedio de duración de cambios entre referencia es de veinte minutos.

- Fiabilidad: en los históricos, cuando este sistema ha corrido la familia de productos en evaluación, ha tenido una disponibilidad promedio de $98 \%$.

- Operarios: en este proceso se utiliza un solo operador. Además de manipular la máquina durante el proceso de refilado, el operador también debe realizar los ajustes operativos requeridos por esta, empacar los productos y preparar o movilizar pallets.

- Inventarios observados: no hay inventarios, pues todos los pallets pasan directamente al siguiente proceso.

- Estaciones: existen cuatro estaciones de trabajo disponibles que se ocupan dependiendo de la carga de trabajo del periodo específico. 
5. Palletizado: los productos ya empacados se embalan sobre estibas y se envuelven en película stretch para proteger la carga. los pallets quedan listas para la expedición y entrega al cliente.

- Equipo: Máquina semiautomática para envolver el pallet con película y protegerla.

- Tiempo de ciclo: el tiempo de ciclo es de 35 minutos por pallet, que contiene una cantidad promedio de setenta rollos. El tiempo de ciclo por rollo es de 0.5 minutos.

- $\quad$ Tiempo de cambio de productos: no aplica.

- Fiabilidad: Con base los históricos, cuando este sistema ha corrido la familia de productos en evaluación, ha tenido una disponibilidad promedio de $98 \%$.

- Operarios: en este proceso se utiliza un solo operador. Además de manipular la máquina durante el proceso de palletizado, también debe realizar los ajustes operativos requeridos por esta y adelantar las movilizaciones de pallets listas para expedición a despachos.

- Inventarios observados: 0,5 días de inventario de producto terminado listo para despachar.

La toma de pedidos realizada en el departamento de Despachos, inicia con la recepción interna, almacenamiento, acopio y despacho de los productos terminados para los clientes o usuarios finales. En la familia de producción estudiada, es el departamento de Despachos quien funge como cliente, dado que este es a quien se le expide diariamente los productos terminados. Asimismo, la demanda mensual alcanza los 116,751 rollos promedio de película plástica para uso general, el cual varían de 84 rollos a 164 rollos, dependiendo de las dimensiones del empaque. El esquema de trabajo operativo para la familia RPUI, se desarrolla considerando la jornada de trabajo correspondiente a 24 horas continúas, incluyendo todas pausas operativas sin afectar la continuidad del proceso productivo.

\section{d. Control de la producción}

Las operaciones de control son apoyadas mediante el sistema de Planificación de Recursos Empresariales (ERP por sus siglas en inglés) con los módulos de ventas, finanzas, producción, costos, compras, almacenes, entre otros. Los aspectos principales del flujo de información se manejan en las siguientes tres etapas de pedidos, programación de producción y abastecimiento de materiales, como se describe a continuación:

1. Pedidos: El departamento de Planeación de la Producción y Control de Inventarios, recibe los pedidos de clientes, el cual son ingresados a diario por el departamento de Ventas. Con base en los balances de inventarios, se envía las órdenes al área de Administración de la Producción, preparando semanalmente un plan base con las órdenes y prioridades. Asimismo, el departamento de Planeación prepara proyecciones a 30, 60 y 90 días.

2. Programación de producción: El área de Administración de la Producción de la planta recibe semanalmente los planes base preparados por Planeación, y elabora los siguientes documentos para el control de la producción: a) Los programas de producción diarios para el proceso de extrusión/embobinado, que es el proceso marcapaso, que distribuye entre las máquinas y secuencia de acuerdo con la lógica para optimizar la eficiencia operativa; b) un programa de expedición diaria de producto terminado para el proceso de refilado y empaque, y c) un plan semanal de acopio de materiales de empaque para la bodega de almacenamiento de la compañía. La bodega de almacenamiento repone una vez al día por camión el inventario de material de empaque que se requiere para el área de extrusión/embobinado. Para el suministro compuestos, el supervisor realiza la revisión de las necesidades del proceso de extrusión/embobinado, e inspecciona los inventarios de material en pellets. Con base en esa información, decide si es necesario arrancar los procesos de dosificación-mezclado y pelletizado.

3. Abastecimiento de materiales: Para los materiales de empaque, el departamento de Compras solicita pedidos semanales a los proveedores, con base en un documento que especifica las carencias de material de empaque, y que recibe semanalmente del área de Administración de la Producción de la planta. Una vez a la semana, los proveedores despachan por camión a la bodega de almacenamiento de la compañía, en promedio, ocho días después de recibida la orden de compra. Para las materias primas, el departamento de Compras hace un pedido mensual a los proveedores con base en un documento que especifica el consumo de materias primas a 30, 60 y 90 días y que recibe del área de Administración de la Producción de la planta. Finalmente, los proveedores despachan una vez cada quince días por camión, directamente a la planta para reponer los tanques y silos de materias primas, treinta días después de recibida la orden de compra.

Seguidamente, con base en los datos obtenidos en observación directa en planta PVC y siguiendo la metodología VSM, la figura 3, muestra el trazado del flujo de valor de la familia de producción RPUI en su estado actual.

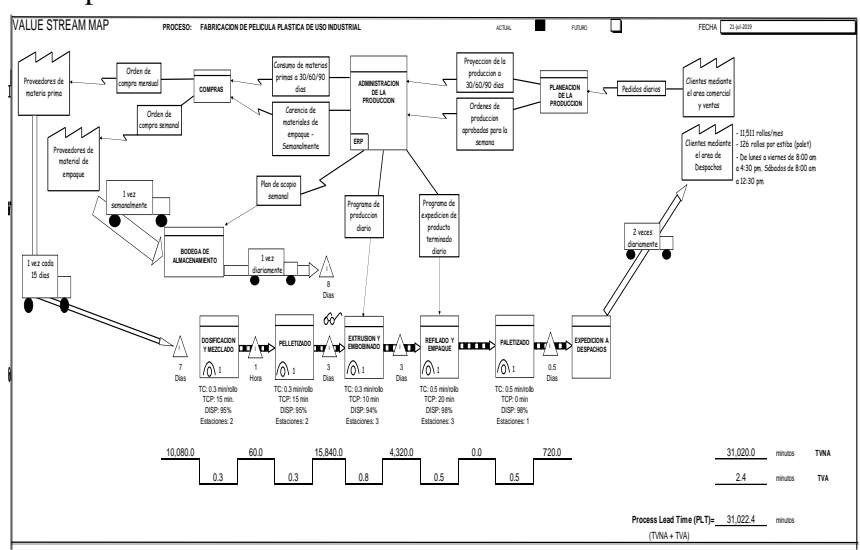

Figura 3: Trazado mapa estado actual planta stretch PVC.

Fuente: Elaboración propia. 
Los tiempos en el proceso total, y el tiempo de valor no agregado en la planta industrial se expresan a continuación:

- $\quad$ Tiempo de proceso total (PLT, process lead time): La sumatoria de los tiempos de procesamiento y las esperas dentro del sistema — desde que ingresa el pedido y atraviesa cada uno de los procesos, hasta que se expide al cliente- es de 31,022.4, equivalente a 21.5 días de tiempo de entrega.

- Tiempo de valor no agregado (TVNA): Las actividades clasificadas como aquellas que no agregan valor (TVNA) suman el 99.99\% del total de tiempo de procesamiento, es decir, 31,020 minutos, el cual comprenden las actividades descritas asi:

4. Inventario de resinas, plastificantes y aditivos: siete días. Se acumulan en silos, tanques y estanterías para asegurar la continuidad de la operación en caso de atrasos por parte de los proveedores.

5. Inventario mezcla o dry blend: una hora. Se mantiene un inventario de mezcla seca previa a ingresar al proceso de pelletizado, con el objetivo de acumular suficientes batches — nombre que se les da en la planta a las cargas de material mezclado-o generar una reserva para garantizar la operación continua de la extrusora de pelletizado.

6. Inventario de pellets: tres días. Su existencia se explica con dos razones principales. La primera es que se debe acumular suficiente producto, con el fin de tener una corrida larga que justifique el tiempo de producción de la extrusora de pelletizado, ya que la fabricación de algunos de los pedidos solo toma un poco más de una hora de producción. La segunda razón es mantener un inventario resorte suficiente para asegurar la continuidad de la operación de extrusión/embobinado, en caso de fallas o incumplimiento presentados en los procesos aguas arriba.

7. Inventario de material de empaque: ocho días. Se trata del tiempo de entrega promedio que tienen los proveedores de los materiales de empaque después de hacerles llegar la orden de compra.

8. Inventario de rollos en maduración: tres días requiere el proceso para que los rollos que salgan del proceso de extrusión/embobinado, puedan eliminar la memoria natural que obtiene el material durante el proceso y logren estabilizar las dimensiones. Es necesario en el proceso para poder garantizar la conformidad de los productos.

9. Acumulación de pallets en espera de expedición: doce horas en promedio, es el tiempo de espera de un pallet hasta que sea recogida por el departamento de Despachos.

La sumatoria de los tiempos de ciclo de los procesos que intervienen en la generación de valor (TVA), constituye un lapso risible respecto al tiempo de proceso total. Indicando, que sin tener en cuenta las esperas listadas antes, a cada unidad de producto le tomaría 2.4 minutos en obtener el valor por el cual el cliente está dispuesto a pagar.

\section{PROPUESTA DE SOLUCIÓN O MEJORAS}

\section{a. Identificación de despilfarros - Mapa de estado futuro}

Seguidamente, se establecen las acciones necesarias para adecuar la familia de producto RPUI a todos los procesos que pertenecen a la planta de producción película PVC. De esta forma se definirá el verdadero flujo continúo teniendo como línea de seguimiento la metodología VSM [64]. Para el trazado del mapa de estado futuro se respondió al siguiente orden de interrogantes:

1. ¿Cuál es el Takt time disponible de los procesos situados más adelante y más cerca del cliente?

El cálculo del Takt time, tiene en cuenta el tiempo disponible de trabajo en la planta de película PVC — dos turnos de 24 horas por día. Los tiempos de descanso y alimentación no afectan la continuidad del proceso productivo, dado que se hacen relevos para mantener las máquinas trabajando. Lo anterior, indica que se cuenta con 1,440 minutos al día para el proceso. Para determinar la demanda se toma en cuenta la venta promedio de un mes expresada en unidades. Se tiene en cuenta el periodo de 2018 (Figura 4). En promedio, se vendieron 45,640 rollos al mes, lo que daría una demanda diaria de 1,541 rollos.

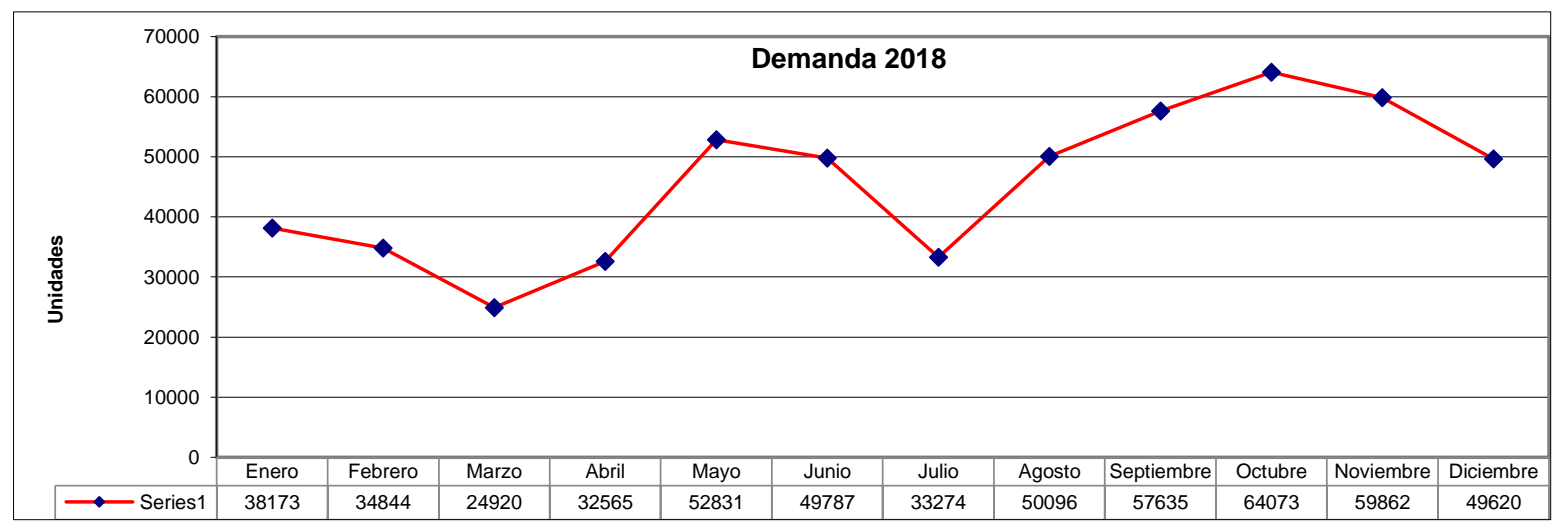

Figura 4: Demanda promedio de producto RPUI planta stretch PVC 2018.

Fuente: Elaboración propia.

Siguiendo la fórmula establecida, el Takt time de la familia de producción RPUI estudiada es de 0.9 minutos/rollo, lo que significa que la planta debe fabricar un rollo cada 0.9 minutos.

$$
\text { Takt time }=\frac{1,440 \text { minutos al día }}{1,541 \text { rollos al día }}=0.9 \text { minutos } / \text { rollo }
$$


2. ¿Se almacenarán los productos terminados en un supermercado para que el cliente los hale, o se entregarán directamente al cliente?

La necesidad de establecer un punto de abastecimiento regulado, para la expedición del producto terminado al cliente, se basa en la variabilidad de la demanda. La planta suministra el producto a una bodega que acopia todos los productos de la empresa, y, desde allí, se despacha a los clientes finales. Como resultado de esto, la demanda se convierte en un flujo estable, con incrementos estacionales bastantes suavizados (ver gráfica de demanda 2018). Otro aspecto a tener en cuenta es el volumen del material producido a diario, con salidas cercanas a los 50 pallets al día que ocupan demasiado espacio y generan la necesidad del retiro del material de manera frecuente. De lo anterior, se concluye que la entrega se manejará directamente al cliente.

3. ¿Qué procesos de la cadena de valor se pueden convertir en flujo continuo (FC)?

La variable a considerar para establecer el FC, debe ser compatible con los tiempos de ciclo de las actividades entre ellas y, a su vez, con el Takt time. Para este análisis se realiza el estudio de los tiempos de ciclo de las actividades que conforman la cadena de valor. A través del siguiente balance de tiempos de ciclo (Figura 5) podemos contrastar la cantidad de tiempo que toma la fabricación de cada rollo con respecto al Takt time.

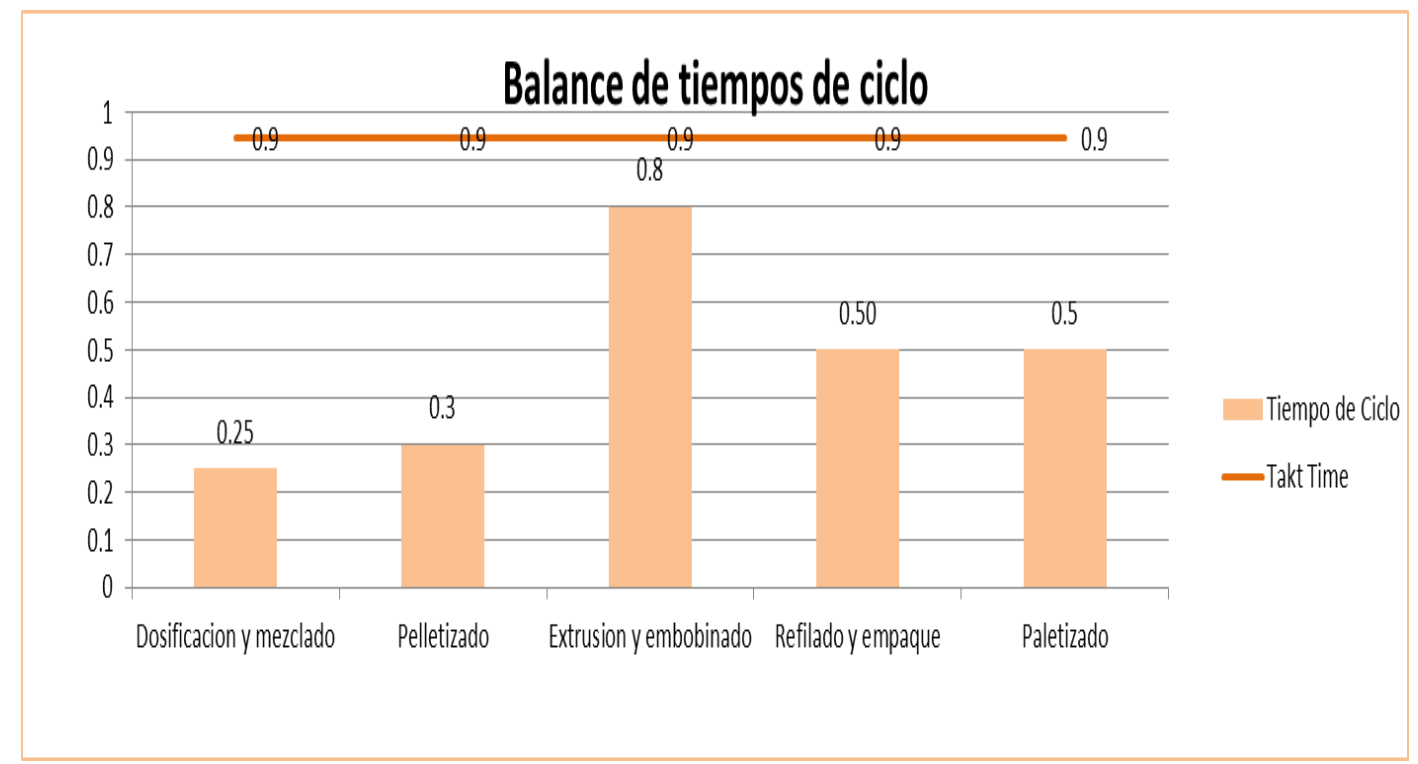

Figura 5: Balance de tiempos de ciclo.

Fuente: Elaboración propia.

La figura 5, presenta que las actividades de dosificación/mezclado y pelletizado, tienen tiempos de ciclo similares, pero, a la vez, muy por debajo del tiempo de Takt time. Adicionalmente, estas etapas del proceso son comunes para las otras familias de producción. En estas actividades no se considerará alinear en flujo continuo con el resto del proceso y, más adelante, se evaluará el establecimiento de supermercado para la alimentación de los procesos aguas abajo; sin embargo, debido a la similitud de tiempos de ciclo entre ellas, es posible fusionar las actividades dosificación/mezclado con pelletizado, creando una línea directa entre ellas. De esta manera, quedará una sola caja de proceso que combina los datos de ciclo entre ambas.

Para el proceso de extrusión y embobinado, ya descontada la capacidad ocupada en las demás familias de producción, el tiempo de ciclo es de 0.8 minutos por rollo, lo cual se acerca al Takt time, pero no se asemeja al tiempo de ciclo de los dos procesos posteriores. También existe la limitante de que, posterior a este proceso, se deben dejar los productos en una etapa de maduración de tres días, necesaria con la tecnología actual, antes de pasar a las actividades siguientes. No obstante, se puede establecer un FC con un retraso equivalente al tiempo requerido para la maduración, para establecer el control de inventario por el sistema PEPS (primeros en entrar, primeros en salir).

Finalizada la etapa de maduración, los rollos pasan a las actividades de refilado y palletizado, siguiendo el orden de llegada de acuerdo con el sistema PEPS. La aplicación de la técnica 5s facilita ordenar las estibas con productos en proceso, y distribuir el trabajo entre las máquinas refiladoras en operación. En este punto se requiere usar la técnica SMED, para lograr disminuir los tiempos de cambio entre referencias y así poder aumentar la disponibilidad de máquina.

4. ¿En qué parte de la cadena de valor se deben establecer supermercados para controlar la producción de procesos anteriores?

A partir del análisis del flujo de materiales de la cadena de valor, y la lógica de comparación de los tiempos de ciclo con el Takt time, se concluye que, para el suministro de compuesto pelletizado al proceso de extrusión y embobinado, se requiere crear un centro de abastecimiento controlado o supermercado entre ambos. Lo que traerá los siguientes beneficios:

- Mejoramiento en tiempo de ciclo del proceso de dosificado/mezclado y pelletizado (0.6 minutos) respecto el tiempo de ciclo anterior (0.8 minutos). El área de pelletizado entregaría para la familia de producción estudiada hasta $33 \mathrm{TM} /$ día, mientras que el proceso de embobinado puede consumir de 17 a $24 \mathrm{TM} /$ día. Este desfase de capacidad hace impráctico tratar de alinear ambos procesos en flujo continuo, y aun así es posible crear un regulador para mantener el principio halar en la cadena de valor. 
- El proceso de dosificado/mezclado y pelletizado también sirve a las otras familias de producción, con el apoyo de un espacio que le permita nivelar su producción de tal manera que se organice la fabricación de las distintas referencias sin caer en ineficiencia operativa.

De esta forma, se elimina la necesidad de tener el inventario de tres días, dado que, al implementar el sistema de halar, únicamente se producirían las cantidades reales que se requieran de forma diaria. Asimismo. es viable implementar un supermercado como abastecimiento regulado de la materia prima al proceso. En este momento no es posible reducir los tiempos de informar oportunamente al departamento de Compras sobre la necesidad de aumentar, disminuir, adelantar o atrasar la llegada de los productos, siempre que se pueda. Un tercer supermercado se establecerá en la recepción de materiales de empaque de la planta, la cual suministrará al proceso de Embobinado, información acerca el control del material y cantidades producidas.

5. ¿Qué punto de la cadena de producción se convertirá en proceso marcapaso (MP)?

El proceso MP a ser programado, es extrusión/embobinado. En razón a que marca el ritmo de la producción, esto permitirá condicionar el trabajo de los procesos ubicados aguas arriba -dosificado/mezclado y pelletizado-, y los procesos aguas abajo, que trabajarán en flujo continuo. Extrusión/embobinado es el proceso que se encuentra inmediatamente después del supermercado que se estableció en el numeral anterior, debido a que con la diferencia de capacidad es imposible establecer un FC en este punto.

6. ¿Cómo se nivelará la combinación de productos en el proceso marcapaso (MP)?

Para reemplazar el modelo actual de programación de la planta, la cual consiste en la secuenciación de los lotes enviados por Planeación y dependen del balance de inventarios que estos realicen con respecto a los pedidos; se tendrá como objetivo conseguir un programa lo suficientemente estable como para lograr un flujo constante a través de la cadena de valor. Esto viabilizará la mejor planificación de las operaciones y la sincronización entre las actividades/procesos y, además, con los proveedores de materiales de empaque, reducirá el tiempo de entrega y disminuirá la necesidad de inventarios en planta al anticipar las cantidades y referencias que se usarán semanalmente mediante la coordinación de entregas diarias. Según lo mencionado, el proceso de extrusión/embobinado tiene cuatro máquinas en operación que se comparten entre todas las familias de producción. Teniendo en cuenta que la producción de familia evaluada, Rollos Para Uso Industrial, corresponde al $71 \%$ del total entregado por la planta, es posible especializar para esta tres de las cuatro máquinas.

Ahora, considerando la clasificación antes mencionada de los productos entre de línea y especiales, se propone inicialmente mantener la programación semanal con actualizaciones diarias, pero relacionando la combinación de productos calculada con base en la demanda diaria, tal como se muestra en la figura 6.

Una vez establecida la mezcla de producto en la fabricación, con los proveedores de material de empaque se procederá a acordar las entregas diarias de los componentes para la ejecución del programa con descarga directa en planta; esto con el fin de alimentar el supermercado sin pasar por la bodega de almacenamiento. De esta manera se disminuye el tiempo de espera, de ocho días a uno, lo que correspondería al inventario de seguridad que se mantendrá en planta y eliminará los movimientos de recepción y trasporte desde la bodega de almacenamiento de materiales.

\begin{tabular}{|l|r|}
\hline Horas/dia & 1,440 \\
\hline Horas/Semana & 10,080 \\
\hline \% Disponibilidad & \\
Extrusión/embobinado & $94 \%$ \\
\hline Horas/Semana Netas de Trabajo & 9,475 \\
\hline
\end{tabular}

\begin{tabular}{|c|c|c|c|c|}
\hline $\begin{array}{l}\text { Tipo de } \\
\text { producto }\end{array}$ & Familia & Suma de Venta Total 2018-2019(KG) & \% Participación & $\begin{array}{c}\text { Product Mix (Horas } \\
\text { semanales) }\end{array}$ \\
\hline Línea & Digi Wrap & 71,134 & $1.44 \%$ & 137 \\
\hline Línea & General Purpose Manual Wrap & $1,688,273$ & $34.28 \%$ & 3,248 \\
\hline Línea & Manual \& Automatic Mushroom wrap & 267,806 & $5.44 \%$ & 515 \\
\hline Línea & Meat Wrap/Automatic Omori \& Ulma Wrapper & 220,191 & $4.47 \%$ & 424 \\
\hline Línea & Meat Wrap/Automatic Wrapper & 203,742 & $4.14 \%$ & 392 \\
\hline Línea & Meat Wrap/Manual Wrapping & $1,297,239$ & $26.34 \%$ & 2,496 \\
\hline Línea & Produce Wrap/Manual \& Automatic Wrapper & 69,357 & $1.41 \%$ & 133 \\
\hline Especial & Blue-Green Laundry Wrap & 26,699 & $0.54 \%$ & 51 \\
\hline Línea & Clear Laundry Wrap & 432,672 & $8.79 \%$ & 832 \\
\hline Especial & Darnel IL (Double layer) Automatic Wrapper & 34,300 & $0.70 \%$ & 66 \\
\hline Línea & Duplo & 610,800 & $12.40 \%$ & 1,175 \\
\hline Especial & Blue Meat Film & 185 & $0.00 \%$ & 0 \\
\hline Especial & Meat Wrap/Automatic Ishida Wrapper & 2,565 & $0.05 \%$ & 5 \\
\hline & Total general & $4,924,964$ & $100 \%$ & 9,475 \\
\hline
\end{tabular}

\begin{tabular}{|l|r|}
\hline \multicolumn{1}{|c|}{ Tipo de producto } & \multicolumn{1}{c|}{$\begin{array}{c}\text { Suma de \% } \\
\text { Participación }\end{array}$} \\
\hline Línea & $98.71 \%$ \\
\hline Especial & $1.29 \%$ \\
\hline
\end{tabular}

Figura 6: Combinación de producto semanal.

Fuente: Elaboración propia. 
7. ¿Qué incremento regular de trabajo se usará como unidad de tiempo para retirar los productos del proceso marcapaso?

La programación actual del MP se realiza con lotes de producción calculados en función del nivel de inventario, dando como resultado corridas más o menos largas que, aunque no tienen un orden específico y no están necesariamente alineadas con la demanda del cliente, optimizan la disponibilidad de la máquina al secuenciar los productos entre similares; esto disminuye en cantidad e impacto a los cambios entre referencias. En este sentido, se evitará un cambio drástico en este aparte; en cambio, se tendrá como tiempo de referencia un día para evaluar la gestión en reemplazo de los programas semanales.

Para nivelar la producción se tendrá como base la técnica heijunka (nivelación de carga), pero ajustada de tal manera que se sirva de los pedidos diarios que informe el departamento de Planeación, y no por lo Kanban de retiro de producto terminado, dadas las características en la organización de la compañía. De esta manera, Administración de la Producción tendrá como fundamento la mezcla de productos realizada en el numeral anterior y, para evaluar la gestión, usará la información de los pedidos diarios, generará un programa cotidiano de producción que, adaptado a la demanda con pequeños ajustes de ser necesarios, incluirían las órdenes para los pedidos de los productos especiales. Una vez que se domine esta organización, se irá allanando el camino para que a futuro se pueda establecer un verdadero FC con la demanda en tiempo real.

8. ¿Qué mejoras a los procesos se necesitarán para que el FV actual se convierta en lo que se proyecta en el mapa de estado futuro?

Para la disminución de los tiempos de entrega del proceso de la familia Rollos Para Uso Industrial de la empresa a continuación, se resumen las siguientes acciones:

- Disminuir los tiempos de cambio entre productos en los procesos de refilado y extrusión/embobinado, mediante la implementación de la técnica Single Minute Exchange of Die (SMED), debido a que, para los trece tipos de producto que conforman la familia estudiada, son muchos SKU y la cantidad de cambios en estos procesos se multiplica, convirtiéndose en una limitante para reducir el tiempo de programación, lograr la fabricación de cada pieza cada día y, como consecuencia, reducir y casi que eliminar la necesidad de mantener inventarios de producto terminado.

- Organizar la disposición, ubicación y entrega de materiales por medio de la implementación de 5s en las áreas de almacenamiento y tránsito de materias primas, material de empaque, productos en proceso y producto terminado. La implementación de esta técnica es vital para el éxito del FC con el sistema primeros en entrar, primeros en salir (PEPS) en el área de maduración, entre extrusión/embobinado y refilado, ya que los productos se estacionarán tres días hasta que puedan pasar al siguiente proceso, en el mismo orden en que llegaron.

- Estabilizar la combinación de producción, en el proceso de extrusión/embobinado, con la creación de ciclos previsibles que permitan la coordinación de las actividades entre los diferentes eslabones de la cadena, y se puedan establecer acuerdos con los proveedores de material de empaque para el suministro diario directo a planta. Con esto se eliminarían los intermediarios y la necesidad de inventarios altos, además de que se acortaría el tiempo de entrega requerido para los pedidos, mediante la implementación de una versión de heijunka adaptada a la operación específica de la planta de película strech PVC.

La figura 7, presenta la versión del proceso con todas las mejoras implementadas, con una reducción casi la mitad, en el tiempo de entrega total, lo que manifiesta una mejora en la organización de la planta y un flujo de información menos complejo. Esto impacta directamente en la promesa de valor que tiene la empresa para con sus clientes, además de la reducción del capital de trabajo al disminuir el tamaño de los inventarios en planta, como se presenta en la tabla 8:

Tabla 8: Comparación de indicadores versión actual y futura.

\begin{tabular}{|l|c|c|c|}
\hline \multicolumn{1}{|c|}{ Indicador } & Actual & Futuro & Mejora \% \\
\hline Tiempos de espera en planta (días) & 21.5 & 12.5 & $42 \%$ \\
\hline Tiempo de proceso (min) & 2.4 & 2.4 & $0 \%$ \\
\hline Plazo de entrega total (días) & 21.5 & 12.5 & $42 \%$ \\
\hline Tiempo de inventario ME (días) & 8 & 1 & $88 \%$ \\
\hline Tiempo de inventario MP (días) & 7 & 7 & $0 \%$ \\
\hline Tiempo de inventario PP (días) & 6.04 & 4.00 & $34 \%$ \\
\hline
\end{tabular}

Fuente: Elaboración propia.

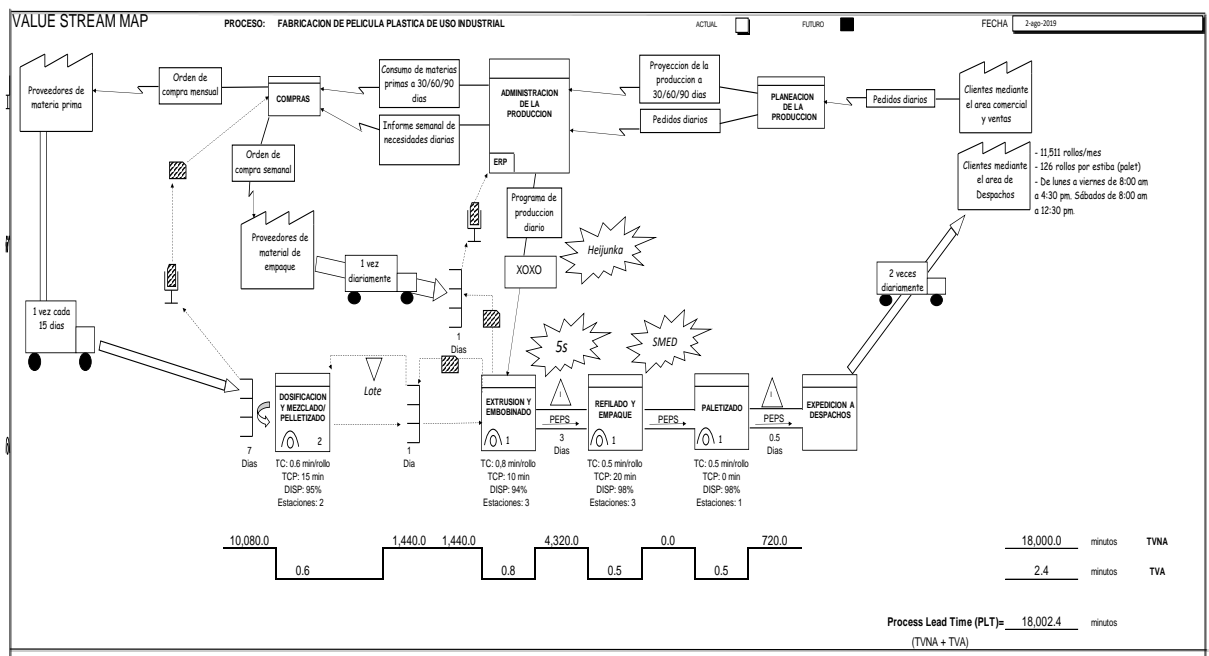

Figura 7: Trazado del mapa de estado futuro planta stretch PVC.

Fuente: Elaboración propia. 
Finalmente, es posible apreciar que, aunque se propone una modificación en el proceso, al fusionar las actividades dosificación/mezclado y pelletizado, los tiempos de este no tienen variación. La inversión requerida para esta fusión no es significativa y el impacto se aprecia en la eliminación del inventario en proceso. Los tiempos mejorados de actividades que no agregan valor (TVNA) fueron los siguientes:

1. Inventario mezcla o dry blend: Una hora menos. Se elimina el inventario en proceso cuando se organizan en serie las actividades de dosificación/mezclado y pelletizado.

2. Inventario de pellets: Dos días menos. Se disminuye la necesidad del alto inventario al implementar el sistema de halado por supermercado. Los requerimientos de pellets se recibirán diariamente y de acuerdo con el consumo del proceso cliente.

Inventario de Material de Empaque: Siete días menos. Al establecer un mix de producción fijo y estable, es posible acordar con el proveedor entregas diarias y directas a la planta. Los materiales se ubicarán en un supermercado donde serán retiradas por el proceso cliente. Se mantiene un día de inventario para evitar interrupción del proceso por eventos imprevistos.

\section{CONCLUSIONES}

La técnica VSM demostró ser una herramienta efectiva para analizar, de forma sistémica, el proceso productivo de la planta industrial de película strech PVC. Esta abarca todas las interrelaciones relevantes en la creación de valor, por lo que viabilizó la identificación de las fuentes de despilfarro e hizo evidentes las acciones a realizar para disminuir el tiempo de entrega a 12.5 días, equivalente a una reducción del $42 \%$, mientras que los días de inventario de producto en proceso se disminuyó en 2.04 días, equivalente a un 34\%, lo que impacta directamente en el capital de trabajo de la unidad de negocio estudiada.

Asimismo, la técnica VSM permite, de manera clara, ver los efectos de la ejecución de acciones focalizadas en puntos específicos del proceso, a lo largo de toda la cadena de valor, y sus resultados. Al ser una representación gráfica de flujos de materiales e información, la herramienta del VSM le permite al investigador recrear las complejidades del sistema y elaborar simulaciones de cambios para evaluar su conveniencia y/o posibles ajustes.

Con la aplicación del VSM para el análisis del proceso de producción, se revela la drástica reducción en el tiempo que le toma a un pedido convertirse en el producto que recibe el cliente, progreso que se obtiene cambiando el flujo de información entre los eslabones de la cadena y eliminando la acumulación de inventarios. Es decir, es posible lograr mejoras significativas en los procesos cuestionando el orden en que estos se llevan a cabo, sin necesidad de incurrir en grandes inversiones o modificar la tecnología existente para la producción.

En general, con la aplicación de la literatura se comprueba la estabilidad y solidez de la técnica VSM desde su origen, siendo un método que ha mostrado pocas variaciones a lo largo de los veinte años desde su primera publicación. Los principales aportes que se han hecho a esta herramienta se refieren al desarrollo de programas informáticos que realizan los cálculos y generan las gráficas. Asimismo, se observa el esfuerzo de algunos autores por conciliar la medición de la efectividad de la técnica con los indicadores de gestión tradicionales (KPI - Key Performance Indicator).

\section{RECOMENDACIONES}

Aunque la técnica VSM demostró ser efectiva, se identificó una posible mejora en adicionar los tiempos que toma el flujo de información para atravesar la cadena de valor. Esta recomendación se sustenta sobre el hecho de que, en la actualidad, la técnica solo contempla la relación de los tiempos que lleva la transformación física de los productos, e ignora aquellas posibles demoras que se presentan en el procesamiento de los pedidos, las órdenes de producción, las compras y la preparación de los programas de producción, dejando por fuera la identificación de otras fuentes de despilfarro.

Para obtener los beneficios de la aplicación del VSM, es necesario que la empresa en estudio se comprometa a ejecutar las acciones establecidas en el plan de trabajo. Se recomienda, de igual manera, continuar con el ejercicio en el resto de la organización para obtener mejoras a nivel corporativo.

Es necesario profundizar en la investigación y el uso de modelos de evaluación, diagnóstico y mejora de procesos en las empresas de la región, en aras de contribuir a la competitividad y al desarrollo económico.

\section{REFERENCIAS}

[1] D. Torres-Reina, “Globalización, empresas multinacionales e historia”. Pensamiento y gestión, Vol. 1, no. 30, p. 165-185, 2011.

[2] ANDI, "Balance 2015 y perspectiva", Asociación Nacional de Industriales, 2016.

[3] L. Garay-Salamanca, “Colombia: Estructura industrial e internacionalización 1967-1996”. Bogotá D. C.: Cargraphics S. A., 1998.

[4] J. Morelos, I. S. Gómez y R. J. De Ávila, "Capacidades de innovación de las pequeñas y medianas empresas del sector metalmecánico en Cartagena, Colombia”. Entramado, Vol. 17, no. 1, p. 12-29, 2021.

[5] ANDI, "Balance 2016 y perspectiva", Asociación Nacional de Industriales, 2017.

[6] T. Ohno, "El Sistema de Producción Toyota Más allá de la producción a gran escala". Nueva York: Routledge, 1991.

[7] J. K. Liker, "The Toyota Way: 14 Management Principles from the World's Greatest Manufacturer Madison": CWL Publishing Enterprises, Inc., 2004.

[8] J. P. Womack, Jones, and D. T. Roos, "La máquina que cambió el mundo". Barcelona: Profit Editorial, 2017.

[9] J. C. Hernández, and A. Vizán, "Lean Manufacturing: Conceptos, técnicas e implantación”. Madrid: Escuela de Organización Industrial - EOI., 2013. 
[10] M. Rajadell, and J. L. Sánchez, "Lean Manufacturing. La evidencia de una necesidad”. Madrid: Ediciones Díaz de Santos., 2010.

[11] A. S. Tejeda, "Mejoras de Lean Manufacturing en los sistemas productivos". Ciencia y sociedad, Vol. 36, no. 2, p. $276-310,2011$.

[12] J. P. Womack, and D. T. Jones, "Lean Thinking". Editorial Planeta, 2012.

[13] M. Carreras, "Lean Manufacturing". La evidencia de una necesidad". Ediciones Díaz de Santos, 2010.

[14] P. Nieto, "Lean Manufacturing": Revisión histórica, 2019.

[15] L. Socconini, "Lean manufacturing. Paso a paso". Marge books, 2019.

[16] M. Poppendieck, "Principles of Lean Thinking". Eden Prairie, 2002.

[17] G. Hodge, K. Goforth, J. Joines, and K. Thoney, "Adapting lean manufacturing principles to the textile industry". Production Planning and Control, Vol. 22, no. 3, p. 237-247, 2011.

[18] A. Morales, J. A. Rojas, L. H. Hernández, A. Morales, y M. Y. Jiménez, "Modelo de un sistema de producción esbelto con redes de Petri para apoyar la toma de decisiones". Ingeniare. Revista Chilena de Ingeniería, Vol. 23, no. 2, p.182-195, 2015.

[19] H. H. González, N. Marulanda y F. J. Echeverry, "Diagnosis in the implementation of Lean manufacturing tools based on the operation strategy in some companies from the textile sector in Colombia: a case report". Revista EAN, Vol. 1, no. 85, p. 199-218, 2018.

[20] E. W. Dickson, S. Singh, D. S. Cheung, C. C. Wyatt, and A. S. Nugent, "Application of lean manufacturing techniques in the emergency department”. The Journal of Emergency Medicine, Vol. 37, no. 2, p. 177-182, 2009.

[21] T. Ohno, "Toyota production system: beyond large-scale production". crc Press, 1998.

[22] Japan Management Association. "KANBAN: Just-in-time en Toyota". Routledge, 2018.

[23] Y. Monden, "Toyota production system: an integrated approach to just-in-time". Productivity Press, 2011.

[24] J. B. Naylor, M. M. Naim, and D. Berry, "Leagility: Integrating the lean and agile manufacturing paradigms in the total supply chain". International Journal of Production Economics, Vol. 62, no. 1, p. 107-118, 1999.

[25] C. Carro, y D. A. González, “Administración de la calidad total”, 2012.

[26] A. Brintrup, D. Ranasinghe, and D. McFarlane, "RFID opportunity analysis for leaner manufacturing”. International Journal of Production Research, Vol. 48, no. 9, p. 2745-2764, 2010.

[27] M. Rother, J. Shook, y Lean Enterprise Institute. "Observar para crear valor: Cartografía de la cadena de valor para agregar valor y eliminar muda”. Brooklin: Lean Enterprise Institute, 1999.

[28] T. Rohac, and M. Januska, "Value Stream Mapping Demonstration on Real Case Study". Procedia Engineering, Vol. 1, no. 100, p. 520529, 2015.

[29] S. J. Pavnaskar, J. K. Gershenson, and A. B. Jambekar, "Classification scheme for lean manufacturing tools". International Journal of Production Research, Vol. 41, no. 13, p. 3075-3090, 2003.

[30] C. Junjie, "The Research of Overproduction Mechanism from the Perspective of Industrial Policies: Empirical Evidence from Manufacturing Industry”. Journal of Finance \& Economics, Vol 1, no 5, p. 1-7, 2016.

[31] W. G. Sullivan, T. N. McDonald, and V. M. Van Aken, "Equipment replacement decisions and lean manufacturing". Robotics and Computer-Integrated Manufacturing, Vol. 18, no. 3, p. 255-265, 2002.

[32] U. Guin, Z. Zhou, and A. Singh, "Robust design-for-security architecture for enabling trust in ic manufacturing and test". Transactions on Very Large Scale Integration (VLSI) Systems, Vol. 26, no. 5, p. 818-830, 2018.

[33] M. Braglia, G. Carmignani, and F. Zammori, "A new value stream mapping approach for complex production systems". International journal of production research, Vol. 44, no. 18, p. 3929-3952, 2006

[34] J. Morelos, T. F. Herrera and E. D. H. Granadillo, "Behaviour of Productivity Indicators and Financial resources in the field of extraction and exploitation of minerals in Colombia". International Journal of Productivity and Quality Management, Vol. 25, no. 3, p. 349-367, 2018.

[35] L. Serrano, y N. R. Ortiz, "Una revisión de los modelos de mejoramiento de procesos con enfoque en el rediseño. Estudios Gerenciales, Vol. 28, no.125, p. 13-22, 2012.

[36] R. Kaplinsky, and M. Morris, “A handbook for value chain research”. Brighton: University of Sussex, Institute of Development Studies, 2000.

[37] C. Grewal, "An initiative to implement lean manufacturing using value stream mapping in a small company". International Journal of Manufacturing Technology and Management, Vol. 15, no. 3, p. 404-417, 2008.

[38] J. Bhamu, J. V. Shailendra-Kumar, and K. S. Sangwan, "Productivity and quality improvement through value stream mapping: a case study of Indian automotive industry". International Journal of Productivity and Quality Management, Vol. 10, no. 3, p. $288-306,2012$.

[39] P. Kuhlang, S. Hempen, W. Sihn, and J. Deuse, "Systematic improvement of value streams-fundamentals of value stream oriented process management”. International Journal of Productivity and Quality Management, Vol. 12, no. 1, p. 1-17, 2013.

[40] W. W. Fisher, S. Barman, and P. L. Killingsworth, Value stream mapping for improving academic advising. International Journal of Information and Operations Management Education, Vol. 4, no. 1, p. 45-59, 2011.

[41] P. Hines, and N. Rich, "The Seven Value Stream Mapping Tools". International Journal of Operations \& Production Management, Vol. 1, no. 17, p. 46-64, 1997.

[42] J. A. Garza, J. T. Romero, K. Govindan, A. Cherrafi, and U. Ramanathan, "A PDCA-based approach to environmental value stream mapping (E-VSM)". Journal of Cleaner Production, Vol.180, no. 1, p. 335-348, 2018.

[43] D. Antonelli, and D. Stadnicka, "Combining factory simulation with value stream mapping: a critical discussion". Procedia CIRP, Vol. 67, no. 1, p. 30-35, 2018.

[44] A. Lugert, A. Batz, and H. Winkler, "Empirical assessment of the future adequacy of value stream mapping in manufacturing industries". Journal of Manufacturing Technology Management, Vol. 29, no. 5, p. 886-906, 2018.

[45] J. Singh, H. Singh, D. Singh, and K. Singh, "Evaluating lean thinking using value stream mapping in manufacturing industry-a case study". International Journal of Productivity and Quality Management, Vol. 22, no.1, p. 101-116, 2017.

[46] M. P. Sarria, G. A. Fonseca, and C. C. Bocanegra, "Modelo metodológico de implementación de Lean Manufacturing". Revista Escuela de Administración de Negocios, Vol. 1, no. 83, p. 51-71, 2017.

[47] F. González, "Manufactura esbelta, principales herramientas". Revista Panorama Administrativo, Vol. 1, no. 1, p. 85-112, 2007.

[48] C. Veres, L. Marian, S. Moica, and K. Al-Akel, "Case study concerning 5S method impact in an automotive company". Procedia Manufacturing, Vol. 22, no. 1, p. 900-905, 2018.

[49] F. C. Filip, and V. Marascu-Klein, "The 5S lean method as a tool of industrial management performances". Materials Science and Engineering, Vol. 95, no. 1, p. 12-127, 2015. 
[50] M. A. Almomani, M. Aladeemy, A. Abdelhadi, and A. Mumani, "A proposed approach for setup time reduction through integrating conventional SMED method with multiple criteria decision-making techniques". Computers and Industrial Engineering, Vol. 66, no. 2, p. 461-469, 2013.

[51] S. Shingo, "A revolution in manufacturing: the SMED system". Routledge, 2019.

[52] J. Matzka, M. Di Mascolo, and K. Furmans, "Buffer sizing of a Heijunka Kanban system”. Journal of Intelligent Manufacturing, Vol. 23, no. 1, p. 49-60, 2012.

[53] P. Korytkowski, T. Wisniewski, and S. Rymaszewski, "Multivariate simulation analysis of production leveling (heijunka)-a case study". IFAC Proceedings Volumes, Vol. 46, no. 9, p. 1554-1559, 2013.

[54] M. Tekin, M. Arslandere, M. Etlioğlu, O. Koyuncuoğlu, and E. Tekin, “An Application of SMED and Jidoka in Lean Production”. In The International Symposium for Production Research, Vol. 1, no. 1, pp. 530-545), 2018.

[55] E. Turanoglu, M. Cakmakci, and C. Kahraman, "Fuzzy Copras method for performance measurement in total productive maintenance: a comparative analysis". Journal of Business Economics and Management, Vol. 17, no. 5, p. 663-684, 2016.

[56] M. Ahmad, D. Dennehy, K. Conboy, and M. Oivo, "Kanban in software engineering: A systematic mapping study". Journal of Systems and Software, Vol. 137, no. 1, p. 96-113, 2018.

[57] D. Mitra, and I. Mitrani, "Analysis of a kanban discipline for cell coordination in production lines, II: Stochastic demands". Operations research, Vol. 39, no. 5, p. 807-823, 1991.

[58] B. Singh, S. K. Garg and S. K. Sharma, "Value stream mapping: literature review and implications for Indian industry". The International Journal of Advanced Manufacturing Technology, Vol. 53, no.5, p. 799-809, 2011.

[59] J. Dal Forno, F. A. Pereira, F. A. Forcellini, and L. M. Kipper, "Value Stream Mapping: a study about the problems and challenges found in the literature from the past 15 years about application of Lean tools". The International Journal of Advanced Manufacturing Technology, Vol. 72, no. 5, p. 779-790, 2014.

[60] E. J. Lourenço, A. J. Baptista, J. P. Pereira, and C. Dias, "Multi-layer stream mapping as a combined approach for industrial processes eco-efficiency assessment”. In Re-engineering Manufacturing for Sustainability, Vol. 1, no. 1, p. 427-433, 2013.

[61] R. Sindhwani, P. L. Singh, R. Chopra, K. Sharma, A. Basu, D. K. Prajapati, and V. Malhotra, "Agility Evaluation in the Rolling Industry: A Case Study". In Advances in Industrial and Production Engineering, Vol. 1, no. 1, p. 753-770, 2019.

[62] Y. H. Lian and H. P. Van-Landeghem, "Analysing the effects of Lean manufacturing using a value stream mapping-based simulation generator". International Journal of Production Research, Vol. 45, no. 13, p. 3037-3058, 2007.

[63] K. Barcia, and C. De Loor, "Metodología para mejorar un proceso de ensamble aplicando el Mapeo de la Cadena de Valor (VSM)". Revista Tecnológica Espol, Vol. 20, no. 1, p. 31-38, 2007.

[64] M. Rother, and J. Shook, "Learning to See: Value Stream Mapping to Add Value and Eliminate Muda". The Lean Enterprise Institute. Inc., Brookline, MA, 1999. 